\title{
ON THE EXISTENCE OF GOOD STATIONARY STRATEGIES
}

\author{
BY \\ WILLIAM D. SUDDERTH( $\left.{ }^{1}\right)$
}

Introduction. A gambler's problem, as formulated by Dubins and Savage in [6] consists of a set $F$ of fortunes, a bounded utility function $u$ on $F$ to the real numbers, and, for each $f$ in $F$, a set $\Gamma(f)$ of gambles (finitely additive probability measures defined on all subsets of $F$ ). A strategy $\sigma$ available in the gambling house $\Gamma$ at the fortune $f$ is a sequence $\sigma_{0}, \sigma_{1}, \ldots$ where $\sigma_{0} \in \Gamma(f)$ and, for each positive integer $n, \sigma_{n}$ is a gamble-valued function defined on $F \times F \times \cdots \times F$ ( $n$-factors) such that $\sigma_{n}\left(f_{1}, \ldots, f_{n}\right) \in \Gamma\left(f_{n}\right)$ for each partial history $\left(f_{1}, \ldots, f_{n}\right)$. The strategy $\sigma$ may be regarded as a probability measure on the infinite product $H=F \times F \times \cdots$ and $\sigma_{n}\left(f_{1}, \ldots, f_{n}\right)$, as the conditional $\sigma$-distribution of $f_{n+1}$ given the first $n$ coordinates are $\left(f_{1}, \ldots, f_{n}\right)$. A gambler with fortune $f$ chooses an available strategy $\sigma$ and a stop rule $t$ and gets a return $u(\sigma, t)$, the expected value of $u\left(f_{t}\right)$ under $\sigma$. By $U(f)$ is denoted the sup $u(\sigma, t)$ taken over all available $\sigma$ and stop rules $t$. $U(f)$ is the best a gambler with fortune $f$ can do.

If a gambler using the strategy $\sigma$ is not allowed to terminate play, he receives $u(\sigma)=\lim \sup _{t \rightarrow \infty} u(\sigma, t) . V(f)$ is the sup $u(\sigma)$ taken over all strategies $\sigma$ available at $f$. If $\Gamma$ is leavable, that is, if the one-point gamble $\delta(f)$ is in $\Gamma(f)$ for all $f$, then $V=U[6$, Corollary 3.3.2, p. 42].

A strategy $\sigma$ is said to be stationary if there is a gamble-valued function $\gamma$ on $F$ such that $\sigma_{n}\left(f_{1}, \ldots, f_{n}\right)=\gamma\left(f_{n}\right)$ for all $n>0$ and each partial history $\left(f_{1}, \ldots, f_{n}\right)$. A family of strategies $\bar{\sigma}$ is a function on $F$ such that $\bar{\sigma}(f)$ is a strategy for each $f$. A family of strategies $\bar{\sigma}$ is stationary if there is a gamble-valued function $\gamma$ on $F$ such that, for each $f, \bar{\sigma}(f)_{0}=\gamma(f)$ and, for each partial history $\left(f_{1}, \ldots, f_{n}\right)$, $\bar{\sigma}(f)_{n}\left(f_{1}, \ldots, f_{n}\right)=\gamma\left(f_{n}\right)$. We write $\gamma^{\infty}$ for the family $\bar{\sigma}$.

Dubins and Savage in [6] raised the question whether stationary families of nearly optimal strategies are available in any leavable gambling house. In particular, they showed that if $F$ is finite and $\Gamma$ is leavable, then, for any $\varepsilon>0$, there is a stationary family $\gamma^{\infty}$ available in $\Gamma$ whose utility $u\left(\gamma^{\infty}(f)\right)$ exceeds $V(f)-\varepsilon$ for all $f$ in $F[6$, p. 58]. Donald Ornstein has extended this result to the case when $F$ is countable and all gambles are countably additive [10, Theorem $\mathrm{B}]$. The theorem is

Received by the editors September 1, 1967.

(1) I am grateful to the National Science Foundation for a Graduate Fellowship from September, 1963 to June, 1967. The research for this doctoral dissertation was subsequently sponsored by the Air Force Office of Scientific Research, Office of Aerospace Research, United States Air Force, under AFOSR Grant AF-AFOSR-1312-67. 
not true if $F$ is uncountable and Ornstein has given a counter-example in which all gambles are countably additive and have countable support [10, Theorem A]. A result obtained here states that, in every leavable gambling house, there is available a stationary family whose utility is within $\varepsilon$ of the best a gambler can do when restricted to a fixed finite number of bets (Theorem 1.1). This result, together with techniques borrowed from [1], [2], [3], and [12], enable me to treat the problem in a Borel measurable setting and establish Theorem 2.3.

Unless otherwise indicated, the terminology and notation of this paper are intended to have the same meaning as in [6].

1. Stationary strategies are enough if $U_{\omega}=U$. For $\Gamma$ a leavable gambling house, $U_{n}(f)$ is defined as in [6] to be the $\sup u(\sigma, t)$ over all strategies $\sigma$ available at $f$ in $\Gamma$ and stop rules $t \leqq n$.

THEOREM 1. Let $\Gamma$ be a leavable gambling house, $n$ be a positive integer and $\varepsilon>0$. Then there is a stationary family $\gamma^{\infty}$ available in $\Gamma$ satisfying

$$
u\left(\gamma^{\infty}(f)\right) \geqq U_{n}(f)-\varepsilon
$$

for all $f$ in $F$. Moreover, $\gamma$ may be chosen so that, for each $f$, the $\gamma^{\infty}(f)$-probability that $\gamma^{\infty}(f)$ stagnates by time $k$ converges to 1 as $k$ approaches $\infty$.

Proof. Let $0=\varepsilon_{0}<\varepsilon_{1}<\cdots<\varepsilon_{n}=\varepsilon$. And define

$$
W(f)=\max _{0 \leqq k \leqq n}\left[U_{k}(f)-\varepsilon_{k}\right]
$$

Here $U_{0}=u$.

If $W(f)=u(f)$, let $\gamma(f)=\delta(f)$.

If $W(f)>u(f)$, choose some $k$ for which $W(f)=U_{k}(f)-\varepsilon_{k}$. Then choose $\gamma(f)$ in $\Gamma(f)$ so that

$$
\gamma(f) U_{k-1} \geqq U_{k}(f)-\alpha
$$

where

$$
\alpha=\frac{1}{2} \min _{1 \leqq k \leqq n}\left(\varepsilon_{k}-\varepsilon_{k-1}\right) .
$$

Then

$$
\begin{aligned}
\gamma(f) W & \geqq \gamma(f) U_{k-1}-\varepsilon_{k-1} \\
& \geqq U_{k}(f)-\frac{1}{2}\left(\varepsilon_{k}-\varepsilon_{k-1}\right)-\varepsilon_{k-1} \\
& =W(f)+\frac{1}{2}\left(\varepsilon_{k}-\varepsilon_{k-1}\right) \\
& \geqq W(f)+\alpha .
\end{aligned}
$$

To prove (1) it suffices to show $u\left(\gamma^{\infty}(f)\right) \geqq W(f)$ for all $f$. This is obvious if $u(f)=W(f)$. If $u(f)<W(f)$, the desired result follows from the two lemmas below. 
LEMma 1. Let $W$ be any bounded, real-valued function defined on $F$ and let $(\sigma, t)$ be any policy. Then, for all $f$,

$$
W(\sigma, t)=W(f)+\int\left[\varepsilon_{0}+\varepsilon_{1}\left(f_{1}\right)+\cdots+\varepsilon_{t(h)-1}\left(f_{1}, \ldots, f_{t(h)-1}\right)\right] d \sigma(h)
$$

where

$$
h=\left(f_{1}, f_{2}, \ldots\right), \quad \varepsilon_{0}=\sigma_{0} W-W(f),
$$

and, for each nonnegative $n$,

$$
\varepsilon_{n}\left(f_{1}, \ldots, f_{n}\right)=\sigma_{n}\left(f_{1}, \ldots, f_{n}\right) W-W\left(f_{n}\right) .
$$

Proof. The proof is by induction on the structure of $f_{t}$.

For any strategy $\sigma$ and history $h$, denote by $t_{\sigma}(h)$ the time at which $\sigma$ stagnates along $h . t_{\sigma}$ is a (possibly) incomplete stop rule [6, p. 44].

LemMA 2. Suppose $W$ is a bounded, real-valued function on $F$ and $\alpha>0$. Let $\sigma$ be a strategy such that, for every partial history $\left(f_{1}, \ldots, f_{n}\right)$, either $u\left(f_{n}\right)=W\left(f_{n}\right)$ and $\sigma$ stagnates at time $n$ along $\left(f_{1}, \ldots, f_{n}, \ldots\right)$ or $\sigma_{n}\left(f_{1}, \ldots, f_{n}\right) W \geqq W\left(f_{n}\right)+\alpha$. Then

$$
u(\sigma) \geqq W(\sigma) \geqq W(f)
$$

and $\sigma\left[t_{\sigma}<n\right] \rightarrow 1$ as $n \rightarrow \infty$.

Proof. If $\varepsilon_{n}$ is defined as in Lemma 1 , then $\varepsilon_{n} \geqq 0$. By (2), $W(\sigma, t) \geqq W(f)$ for every stop rule $t$. Hence, $W(\sigma) \geqq W(f)$.

Now let $B=\sup |W|$. By Lemma 1 ,

$$
\begin{aligned}
2 B & \geqq W(\sigma, n)-W(f) \\
& =\int\left[\varepsilon_{0}+\varepsilon_{1}\left(f_{1}\right)+\cdots+\varepsilon_{n-1}\left(f_{1}, \ldots, f_{n-1}\right)\right] d \sigma(h) \\
& \geqq \int_{\left[t_{\sigma} \geqq n\right]} n \alpha d \sigma=n \alpha \sigma\left[t_{\sigma} \geqq n\right] .
\end{aligned}
$$

Hence, $\sigma\left[t_{\sigma} \geqq n\right] \leqq 2 B / n \alpha \rightarrow 0$ as $n \rightarrow \infty$.

Also, if $t \geqq n$,

$$
\begin{aligned}
W(\sigma, t)-u(\sigma, t) & =\int\left[W\left(f_{t}\right)-u\left(f_{t}\right)\right] d \sigma \\
& =\int_{\left[t_{\sigma} \geqq t\right]}\left[W\left(f_{t}\right)-u\left(f_{t}\right)\right] d \sigma \\
& \leqq \int_{\left[t_{\sigma} \geqq n\right]} 2 B d \sigma \\
& \leqq \frac{4 B^{2}}{n} \rightarrow 0 \quad \text { as } n \rightarrow \infty .
\end{aligned}
$$

This completes the proof of Theorem 1 . 
Since $U_{\omega}(f)=\sup _{n} U_{n}(f)$, an immediate corollary is

COROLlaRY 1. If $\Gamma$ is leavable, $\varepsilon>0$, and $f$ in $F$, then there is a stationary family $\gamma^{\infty}$ available in $\Gamma$ such that $u\left(\gamma^{\infty}(f)\right) \geqq U_{\omega}(f)-\varepsilon$.

The corollary is of interest since in many important cases $U_{\omega}=U[6$, Theorem 2.15.5, p. 35].

The technique used to prove Theorem 1 can be modified to give

THEOREM 2. Suppose $\Gamma$ is leavable, $n$ is a positive integer, $0<\alpha<1$, and $u \geqq 0$. Then there is a stationary family $\gamma^{\infty}$ available in $\Gamma$ satisfying

$$
u\left(\gamma^{\infty}(f)\right) \geqq \alpha U_{n}(f) \text { for all } f \text { in } F .
$$

An example shows that the strategies $\gamma^{\infty}(f)$ cannot be chosen so as to stagnate "almost surely" as they were in Theorem 1 .

EXAMPLE. Let $F=\{0,1,2, \ldots\} ; \Gamma(0)=\{\delta(0)\}, \Gamma(n)=\left\{\delta(n), p_{n} \delta(n+1)+\left(1-p_{n}\right) \delta(0)\right\}$ for $n>0$ where $0<p_{n}<1$ and their product $p_{1} p_{2} \cdots$ is strictly positive; $u(0)=1$, $u(n)=0$ for $n>0$. Any stationary strategy which satisfies (4) must have $\gamma(n) \neq \delta(n)$ for $n>0$ and hence $\gamma^{\infty}(n)$ never stagnates with probability $p_{n} \cdot p_{n+1} \cdots$.

2. Measurable gambling houses. Let $X$ be a Borel set, which in this note means a Borel subset of a complete separable metric space. Let $\mathscr{B}(X)$ be the $\sigma$-field of Borel subsets of $X$. Denote by $\mathscr{P}(X)$ the set of all countably additive probability measures on $\mathscr{B}(X)$, and let $\Sigma(X)$ be the smallest $\sigma$-field of subsets of $\mathscr{P}(X)$ which makes $p \rightarrow p(B)$ a measurable function of $p$ for each $B$ in $\mathscr{B}(X)$. Then $\mathscr{P}(X)$ is a Borel set with Borel subsets $\Sigma(X)$ (Dubins and Freedman, [5]).

Lemma 1. Let $X$ and $Y$ be Borel sets, $C$ a measurable subset of the product $X \times Y$, and $\psi$ a measurable function from $X$ to $Y$. Then $\{x:(x, \psi(x)) \in C\}$ is a measurable subset of $X$.

Proof. The map $x \rightarrow(x, \psi(x))$ is measurable from $X$ to $X \times Y$.

Roger Purves pointed out this proof to me.

Lemma 1 will be applied several times without specific reference.

Now let $X$ and $Y$ be Borel sets and $p \in \mathscr{P}(X \times Y)$. Let $p_{0}$ be the $p$-marginal distribution of $x$ and let $p_{1}$ be a version of the regular conditional $p$-distribution of $y$ given $x$. Notice $p_{1}$ is a measurable map from $X$ to $\mathscr{P}(Y)$ and, if $q_{1}$ is any other version of the regular conditional $p$-distribution of $y$ given $x$, then $p_{1}(x)=q_{1}(x)$ $p_{0}$-almost certainly. Thus there is a natural map $\phi: \mathscr{P}(X \times Y) \rightarrow \mathscr{P}(\mathscr{P}(Y))$ given by

$$
\phi(p)(A)=p_{0}\left\{x: p_{1}(x) \in A\right\}
$$

for $p$ in $\mathscr{P}(X \times Y)$ and $A$ in $\Sigma(Y)$.

LEMMA 2. $\phi$ is measurable. 
Proof. We need to show $p \rightarrow \phi(p)(A)$ is measurable in $p$ for each $A$ in $\Sigma(Y)$. It is enough to consider $A$ of the form $\{p: p(B)>r\}$ where $B$ is in $\mathscr{B}(Y)$. Hence, it suffices to show $p \rightarrow \int \psi\left(p_{1}(x)(B)\right) d p_{0}(x)$ is measurable for $B$ in $\mathscr{B}(Y)$ and $\psi$ continuous on $[0,1]$.

Let $\mathscr{A}_{n}$ be a finite $\sigma$-field of subset of $X$ for $n=1,2, \ldots$ and choose the $\mathscr{A}_{n}$ so that their union generates $\mathscr{B}(X)$. Denote by $p^{n}(x)$ the conditional $p$-distribution of $y$ given $\mathscr{A}_{n}$. It is easy to see that $p \rightarrow \int \psi\left(p^{n}(x)(B)\right) d p_{0}(x)$ is measurable in $p$. Since $p^{n}(x)(B) \rightarrow p_{1}(x)(B) p_{0}$-almost certainly, as the martingale convergence theorem implies, the proof is complete.

Corollary 1. Let $C$ be a measurable subset of $X \times \mathscr{P}(Y)$. Then the map $p \rightarrow$ $p_{0}\left\{x:\left(x, p_{1}(x)\right) \in C\right\}$ is measurable from $\mathscr{P}(X \times Y)$ to the Borel line.

Proof. Apply Lemma 2 to prove the case of $C$ a measurable rectangle and extend to arbitrary measurable sets.

According to Strauch [11], a gambling house $\Gamma$ is measurable if $F$ is a Borel set, every gamble available in $\Gamma$ is countably additive when restricted to $\mathscr{B}(F)$, and the set $\Gamma^{1}=\{(f, \gamma): \gamma \in \Gamma(f)\}$ is a measurable subset of $F \times \mathscr{P}(F)$. Each gamble $\gamma$ is identified with its restriction to $\mathscr{B}(F)$. In this section, the utility function $u$ is assumed to be bounded and measurable from $F$ to the Borel line.

Let $F^{n}=F \times F \times \cdots \times F$ ( $n$-factors). A measurable strategy $\sigma$ is a sequence $\sigma_{0}, \sigma_{1}, \ldots$ where $\sigma_{0}$ is in $\mathscr{P}(F)$ and, for $n>0, \sigma_{n}$ is a measurable map from $F^{n}$ to $\mathscr{P}(F)$. The finite sequence $\sigma_{0}, \ldots, \sigma_{n-1}$, or $\sigma^{n}$ for short, is a measurable $n$-day strategy. Any measurable strategy $\sigma$ determines a probability measure $p(\sigma)$ on the Borel subsets of $H$, where $H=F \times F \times \cdots$ is given the product Borel structure. Specifically, the $p(\sigma)$-distribution of $f_{1}$ is $\sigma_{0}$ and, for $n>0$, the conditional $p(\sigma)$ distribution of $f_{n+1}$ given $\left(f_{1}, \ldots, f_{n}\right)$ is $\sigma_{n}\left(f_{1}, \ldots, f_{n}\right)$. Similarly, a measurable $n$-day strategy $\sigma^{n}$ determines a probability measure $p\left(\sigma^{n}\right)$ on $\mathscr{B}\left(F^{n}\right)$. We sometimes write $\sigma$ for $p(\sigma)$ and $\sigma^{n}$ for $p\left(\sigma^{n}\right)$ if there is no danger of ambiguity.

For $n>1$ and $f$ in $F$, let $\Gamma^{n}(f)$ be the collection of all $p\left(\sigma^{n}\right)$ where $\sigma^{n}$ is a measurable $n$-day strategy available at $f$ in $\Gamma$ and set

$$
\Gamma^{n}=\left\{(f, p) \in F \times \mathscr{P}\left(F^{n}\right): p \in \Gamma^{n}(f)\right\} .
$$

Define $\Gamma^{\infty}(f)$ to be all $p(\sigma)$ where $\sigma$ is a measurable strategy at $f$ and define $\Gamma^{\infty}$ by analogy with $\Gamma^{n}$.

When $\Gamma$ is nonleavable, it may happen that there is no measurable map $\alpha$ from $F$ to $\mathscr{P}(F)$ such that $\alpha(f) \in \Gamma(f)$ for all $f$ (see p. 16 of [9]). In this case, $\Gamma^{n}$ is empty for $n>1$.

THEOREM 1. $\Gamma^{n}$ is a Borel subset of $F \times \mathscr{P}\left(F^{n}\right)$ for $n=1,2, \ldots$ and $\Gamma^{\infty}$ is a Borel subset of $F \times \mathscr{P}(H)$.

Proof. By the remark above, we can assume that a measurable selector $\alpha$ does exist. Then

$$
\Gamma^{2}=\left\{(f, p) \in F \times \mathscr{P}\left(F^{2}\right): p_{0} \in \Gamma(f), p_{0}\left\{f_{1}: p_{1}\left(f_{1}\right) \in \Gamma\left(f_{1}\right)\right\}=1\right\}
$$


where $p_{1}\left(f_{1}\right)$ is a regular conditional $p$-distribution of $f_{2}$ given $f_{1}$. For if $(f, p)$ is in the right hand set, let $\sigma_{0}=p_{0}$ and

$$
\begin{aligned}
\sigma_{1}\left(f_{1}\right) & =p_{1}\left(f_{1}\right) & \text { if } p_{1}\left(f_{1}\right) \in \Gamma\left(f_{1}\right), \\
& =\alpha\left(f_{1}\right) & \text { if } p_{1}\left(f_{1}\right) \notin \Gamma\left(f_{1}\right) .
\end{aligned}
$$

Let $\sigma^{2}=\sigma_{0}, \sigma_{1}$. Plainly, $\sigma^{2}$ is a 2-day strategy available at $f$ and $p\left(\sigma^{2}\right)=p$. Hence, $(f, p) \in \Gamma^{2}$. The opposite inclusion is obvious. By (1) and Corollary $1, \Gamma^{2}$ is measurable.

Similarly, for $n>2$,

$$
\Gamma^{n}=\left\{(f, p) \in F \times \mathscr{P}\left(F^{n}\right): p_{0} \in \Gamma(f), p_{0}\left\{f_{1}: p_{n-1}\left(f_{1}\right) \in \Gamma^{n-1}\left(f_{1}\right)\right\}=1\right\},
$$

where $p_{n-1}\left(f_{1}\right)$ is a regular conditional $p$-distribution of $\left(f_{2}, \ldots, f_{n}\right)$ given $f_{1}$. Using induction and Corollary 1 , we have proved the first half of the theorem.

Since

$$
\Gamma^{\infty}=\left\{(f, p) \in F \times \mathscr{P}(H):\left(f, p_{n}\right) \in \Gamma^{n}, n=1,2, \ldots\right\}
$$

where $p_{n}$ is the $p$-marginal distribution of $\left(f_{1}, \ldots, f_{n}\right)$, the proof is complete.

Now let

$$
Q_{n}(f)=\sup _{p \in \Gamma^{n}(f)} \int u\left(f_{n}\right) d p
$$

for $f$ in $F$ and $n=1,2, \ldots$.

Recall that a function is absolutely measurable if it is measurable with respect to the completion of every measure on the Borel sets.

TheOREM 2. Let $\Gamma$ be a leavable, measurable house. Then $Q_{n}=U_{n}$ and $U_{n}$ is absolutely measurable for $n=1,2, \ldots$

Proof. The set $\left\{f: Q_{n}(f)>r\right\}$ is the projection of the Borel set

$$
\left\{(f, p) \in \Gamma^{n}: \int u\left(f_{n}\right) d p>r\right\}
$$

for any real $r$. Hence, it is analytic and, therefore, absolutely measurable [7, p. 391].

Now $Q_{1}=U_{1}$. Assume inductively that $Q_{n}=U_{n}$. Let $\varepsilon>0$ and $f_{0} \in F$. It suffices to show $Q_{n+1}\left(f_{0}\right)>U_{n+1}\left(f_{0}\right)-\varepsilon$.

Choose $\sigma_{0}$ in $\Gamma\left(f_{0}\right)$ such that $\sigma_{0} Q_{n}=\sigma_{0} U_{n}>U_{n+1}\left(f_{0}\right)-\varepsilon / 2$. Let

$$
S=\left\{(f, p) \in \Gamma^{n}: \int u\left(f_{n}\right) d p>U_{n}(f)-\varepsilon / 2\right\}
$$

By Theorem 6.3 of [8], there is a measurable map $\bar{\sigma}$ from $F$ to $\mathscr{P}\left(F^{n}\right)$ such that $\sigma_{0}\left\{f: \bar{\sigma}(f) \in S_{f}\right\}=1$. Define a measurable $n+1$-day strategy $\sigma$ available at $f_{0}$ to 
have initial gamble $\sigma_{0}$ and conditional $n$-day strategies $\sigma\left[f_{1}\right]$ satisfying $p\left(\sigma\left[f_{1}\right]\right)$ $=\bar{\sigma}\left(f_{1}\right)$ if $\bar{\sigma}(f) \in S_{f}$. Then

$$
\begin{aligned}
Q_{n+1}\left(f_{0}\right) & \geqq u(\sigma, n+1) \\
& =\int u\left(f_{n}\right) d \bar{\sigma}\left(f_{n} \mid f\right) d \sigma_{0}(f) \\
& \geqq \int U_{n} d \sigma_{0}-\varepsilon / 2 \\
& >U_{n+1}\left(f_{0}\right)-\varepsilon .
\end{aligned}
$$

Corollary 2 (STRAuch [11]). If $\Gamma$ is a measurable house, then $U$ is absolutely measurable.

Proof. We can assume without loss of generality that $\Gamma$ is leavable. Since the $U_{n}$ are absolutely measurable $U=U_{\omega}=\lim _{n \rightarrow \infty} U_{n}$ by Theorem $2.15 .5(\mathrm{~g})$ of [6].

A policy $(\sigma, t)$ is measurable if $\sigma$ is a measurable strategy and $t$ is a stop rule measurable with respect to the product $\sigma$-field on $H$. Strauch asserted in [11] that nearly optimal measurable policies are available in any measurable house $\Gamma$. That such policies are available for leavable $\Gamma$, even with $t$ 's of the form $t \equiv n$, follows from Theorem 2. It is not difficult to show that good measurable policies are available in any measurable house for which there is a Borel map $\alpha: F \rightarrow \mathscr{P}(F)$ with $\alpha(f)$ in $\Gamma(f)$ for all $f$. If there is no such map, then, as was pointed out in the remark preceding Theorem 1, there are no measurable strategies available. However, there do exist nearly optimal measurable policies $(\sigma, t)$ such that $\sigma$ is available in $\Gamma$ until time $t$. That is, for every partial history $\left(f_{1}, \ldots, f_{n}\right), \sigma_{n}\left(f_{1}, \ldots, f_{n}\right) \in \Gamma\left(f_{n}\right)$ if $t\left(f_{1}, \ldots, f_{\dot{n}}, \ldots\right)<n$. This can be derived by passing to the leavable closure and using Theorem 2 or the next theorem.

A stationary family $\gamma^{\infty}$ is measurable if $\gamma$ is a measurable function from $F$ to $\mathscr{P}(F)$. The rest of the paper is devoted to proving

THeOREM 3. Let $\Gamma$ be a leavable, measurable gambling house on $F$. Then for each countably additive probability measure $p$ on the Borel subsets of $F$ and each $\varepsilon>0$, there is available a measurable stationary family $\gamma^{\infty}$ such that

$$
p\left\{f: u\left(\gamma^{\infty}(f)\right) \geqq U(f)-\varepsilon\right\}=1 .
$$

Theorem 3 and the methods used in its proof owe much to previous work in [1], [2], [3], and [12].

An example of Strauch [11] shows that $U$ need not be Borel measurable. The same example therefore shows that Borel measurable stationary families which are everywhere $\varepsilon$-optimal need not exist.

3. Three lemmas. This section is devoted to three lemmas which are needed in the sequel. 
The first lemma formalizes an idea used by Donald Ornstein. Let $\sigma$ be a strategy available at $f$ in some gambling house $\Gamma$. For each history $h=\left(f_{1}, f_{2}, \ldots\right)$ and positive integer $n$, let $p_{n}(h)=\left(f_{1}, f_{2}, \ldots, f_{n}\right)$ denote the $n$-day partial history. Then define a (possibly) incomplete stop rule $t_{\varepsilon}$ to be the first time the gambler reaches a fortune at which the conditional $\sigma$-strategy is not $\varepsilon$-optimal. That is,

$$
\begin{aligned}
t_{\varepsilon}\left(f_{1}, f_{2}, \ldots\right) & =\text { least } n \text { (if any) for which } u\left(\sigma\left[p_{n}(h)\right]\right)<V\left(f_{n}\right)-\varepsilon \\
& =\infty \text { if no such } n \text { exists. }
\end{aligned}
$$

LEMMA 1. Let $\varepsilon>0$ and $t$ be any stop rule. If $u(\sigma)>V(f)-\varepsilon^{2}$, then $\sigma\left[t_{\varepsilon} \leqq t\right] \leqq \varepsilon$.

Proof. One can check that the set $\left[t_{\varepsilon} \leqq t\right]$ is determined by time $t \wedge t_{\varepsilon}$ and is thus finitary [6, p. 15].

According to formula (1) $[6$, p. 42$]$

$$
\begin{aligned}
u(\sigma) & =\int u\left(\sigma\left[p_{t \wedge t_{\varepsilon}}\right]\right) d \sigma \\
& =\int_{\left[t_{\varepsilon}>t\right]} u\left(\sigma\left[p_{t}\right]\right) d \sigma+\int_{\left[t_{\varepsilon} \leqq t\right]} u\left(\sigma\left[p_{t_{\varepsilon}}\right]\right) d \sigma \\
& \leqq \int_{\left[t_{\varepsilon}>t\right]} V\left(f_{t}\right) d \sigma+\int_{\left[t_{\varepsilon} \leqq t\right]}\left(V\left(f_{t_{\varepsilon}}\right)-\varepsilon\right) d \sigma \\
& \leqq \int V\left(f_{t \wedge t_{\varepsilon}}\right) d \sigma-\varepsilon \sigma\left[t_{\varepsilon} \leqq t\right] \\
& \leqq V(f)-\varepsilon \sigma\left[t_{\varepsilon} \leqq t\right] .
\end{aligned}
$$

The last inequality is, of course, because $V$ is excessive for $\Gamma[6$, p. 41].

Since $u(\sigma)>V(f)-\varepsilon^{2}$ by assumption, the proof is complete.

Suppose now that a gambler with fortune $f$ uses a strategy $\sigma$ until some time $t^{\prime}$. And having experienced a partial history $p_{t^{\prime}}(h)=\left(f_{1}, f_{2}, \ldots, f_{t^{\prime}(h)}\right)$ he then changes to a new strategy $\sigma^{\prime}\left(p_{t^{\prime}(h)}\right)$. One might expect if $\sigma$ is a nearly optimal strategy and if the strategies $\sigma^{\prime}(p)$ are nearly optimal that this new composed strategy is also nearly optimal. The next lemma shows that this expectation is fulfilled.

Let $\sigma$ be a strategy and $\sigma^{\prime}$ be a function from partial histories to strategies. If $t^{\prime}$ is any (possibly) incomplete stop rule, define a new strategy $\bar{\sigma}$ called the composition of $\sigma$ with $\sigma^{\prime}$ at time $t^{\prime}$ by letting $\bar{\sigma}_{n}\left(f_{1}, \ldots, f_{n}\right)$ be $\sigma_{n}\left(f_{1}, \ldots, f_{n}\right), \sigma_{0}^{\prime}\left(p_{t^{\prime}(h)}\right)$, or $\sigma_{n-t^{\prime}(h)}^{\prime}\left(p_{t^{\prime}(h)}\right)\left(f_{t^{\prime}(h)+1}, \ldots\right)$ according as $n$ is less than, equal to, or greater than $t^{\prime}(h)$ (cf. [6, p. 22]).

LEMMA 2. Let $\sigma$ be available at $f$ in $\Gamma$ and $\sigma^{\prime}\left(f_{1}, \ldots, f_{n}\right)$ be available at $f_{n}$ in $\Gamma$ for each partial history $\left(f_{1}, \ldots, f_{n}\right)$. Suppose $u(\sigma)>V(f)-\varepsilon^{2}$ and $u\left(\sigma^{\prime}\left(f_{1}, \ldots, f_{n}\right)\right)$ $>V\left(f_{n}\right)-\varepsilon^{\prime}$ whenever $t^{\prime}\left(f_{1}, f_{2}, \ldots, f_{n}, \ldots\right)=n$. If $\bar{\sigma}$ is the composition of $\sigma$ with $\sigma^{\prime}$ at time $t^{\prime}$, then

$$
u(\sigma) \geqq V(f)-\left(\varepsilon^{2}+\varepsilon M+\varepsilon^{\prime}\right)
$$

where $M=\sup \{|u(f)|: f \in F\}$. 
Proof. Let $t$ be any stop rule. It suffices to find a stop rule $\bar{t} \geqq t$ such that

$$
u(\bar{\sigma}, \bar{t}) \geqq V(f)-\left(\varepsilon^{2}+\varepsilon M+\varepsilon^{\prime}\right) .
$$

Since $u(\sigma)>V(f)-\varepsilon^{2}$, we can find a stop rule $t_{1} \geqq t$ with

$$
\sigma\left\{h: u\left(f_{t_{1}(h)}\right)>V\left(f_{t_{1}(h)}\right)-\varepsilon\right\} \geqq 1-\varepsilon .
$$

And for each $h$ such that $t^{\prime}(h)<\infty$, we can find a stop rule $t\left(p_{t^{\prime}(h)}\right)$ with

and

$$
t^{\prime}(h)+t\left(p_{t^{\prime}(h)}\right)\left(f_{t^{\prime}(h)+1}, f_{t^{\prime}(h)+2}, \ldots\right) \geqq t(h)
$$

Define

$$
u\left(\sigma^{\prime}\left(p_{t^{\prime}(h)}\right), t\left(p_{t^{\prime}(h)}\right)\right)>V\left(f_{t^{\prime}(h)}\right)-\varepsilon^{\prime}
$$

$$
\begin{aligned}
\bar{t}(h) & =t_{1}(h) & & \text { if } t_{1}(h) \leqq t^{\prime}(h) \\
& =t^{\prime}(h)+t\left(p_{t^{\prime}(h)}\right)\left(f_{t^{\prime}(h)+1}, \ldots\right) & & \text { if } t_{1}(h)>t^{\prime}(h) .
\end{aligned}
$$

Then $\bar{t} \geqq t$ and

$$
\begin{aligned}
u(\bar{\sigma}, \bar{t}) & =\int u\left(f_{t}\right) d \bar{\sigma} \\
& =\int_{\left[t_{1} \leqq t^{\prime}\right]} u\left(f_{t_{1}}\right) d \sigma+\int_{\left[t_{1}>t^{\prime}\right]} u\left(\sigma^{\prime}\left(p_{t^{\prime}}\right), t\left(p_{t^{\prime}}\right)\right) d \sigma \\
& \geqq \int_{\left[t_{1} \leqq t^{\prime}\right]} V\left(f_{t_{1}}\right) d \sigma-\varepsilon M+\int_{\left[t_{1}>t^{\prime}\right]} V\left(f_{t^{\prime}}\right) d \sigma-\varepsilon^{\prime} \\
& =V\left(\sigma, t_{1} \wedge t^{\prime}\right)-\varepsilon M-\varepsilon^{\prime} \\
& \geqq V(f)-\varepsilon^{2}-\varepsilon M-\varepsilon^{\prime} .
\end{aligned}
$$

Let $\sigma$ and $\sigma^{\prime}$ be strategies. For any nonnegative integer $n, \sigma$ and $\sigma^{\prime}$ agree along $\left(f_{1}, \ldots, f_{n}, \ldots\right)$ up to time $n$ if $\sigma_{0}=\sigma_{0}^{\prime}$ and $\sigma_{k}\left(f_{1}, \ldots, f_{k}\right)=\sigma_{k}^{\prime}\left(f_{1}, \ldots, f_{k}\right)$ for all $k \leqq n$.

Let $g$ be a function on $H$ and $t$ be a stop rule. $g$ is determined by time $t$ if whenever $t(h)=t\left(h^{\prime}\right)$, then $g(h)=g\left(h^{\prime}\right)[6$, p. 15].

LEMMA 3. Suppose $\sigma$ and $\sigma^{\prime}$ are strategies which agree along every history $h$ up to time $t(h)$ for a given stop rule $t$. If $g$ is a bounded function from $H$ to the real numbers and $g$ is determined by time $t$, then

$$
\int g d \sigma=\int g d \sigma^{\prime}
$$

Proof. The proof is by induction on the structure of $g$ and uses formula 2.8.1 of $[6$, p. 17].

If $\sigma$ and $\sigma^{\prime}$ are two strategies for which $\sigma_{0}=\sigma_{0}^{\prime}$, then they stop agreeing at time $\hat{t}$, where $\hat{t}$ is the (possibly) incomplete stop rule defined by

$$
\begin{aligned}
\hat{t}(h) & =\text { least } n \text { (if any) for which } \sigma_{n}\left(f_{1}, \ldots f_{n}\right) \neq \sigma_{n}^{\prime}\left(f_{1}, \ldots, f_{n}\right) \\
& =\infty \text { if no such } n \text { exists. }
\end{aligned}
$$


COROllaRY 1. Let $\sigma$ and $\sigma^{\prime}$ be strategies which stop agreeing at time $\hat{t}$. If $t$ is any stop rule, then

$$
\sigma[t \geqq \hat{t}]=\sigma^{\prime}[t \geqq \hat{t}]
$$

And if $g$ is any bounded function on $H$ which is determined by time $t$, then

$$
\left|\int g d \sigma-\int g d \sigma^{\prime}\right| \leqq \sigma[t \geqq \hat{t}](\sup g-\inf g) \text {. }
$$

In particular,

$$
\left|u(\sigma, t)-u\left(\sigma^{\prime}, t\right)\right| \leqq \sigma[t \geqq \hat{t}](\sup u-\inf u)
$$

Proof. (1) is immediate from Lemma 3. To prove (2), let

$$
\begin{aligned}
g_{0}(h) & =g(h) & & \text { if } t(h)<\hat{t}(h), \\
& =0 & & \text { if } t(h) \geqq \hat{t}(h) .
\end{aligned}
$$

Then $g_{0}$ is determined by time $t \wedge \hat{t}$. So by Lemma 3 ,

$$
\int_{[t<\hat{t}]} g_{0} d \sigma=\int_{[t<\hat{t}]} g_{0} d \sigma^{\prime}
$$

Hence,

$$
\left|\int g d \sigma-\int g d \sigma^{\prime}\right|=\left|\int_{[t \geqq \hat{t}]} g d \sigma-\int_{[t \geqq \hat{t}]} g d \sigma^{\prime}\right|,
$$

and (2) follows from (1).

COROllary 2. Let $\sigma$ and $\sigma^{\prime}$. be strategies which stop agreeing at time $\hat{t}$. Suppose there is some $\varepsilon \geqq 0$ such that for every stop rule $t, \sigma[t \geqq \hat{t}] \leqq \varepsilon$. Then

$$
\left|u(\sigma)-u\left(\sigma^{\prime}\right)\right| \leqq \varepsilon(\sup u-\inf u) .
$$

4. Countably parametrized measurable houses. In proving Theorem 2.3 , it is convenient to consider first a house $\Gamma$ for which $\Gamma(f)$ is countable for each $f$ and then to reduce the general problem to this special case. So let us assume that $\Gamma$ is a measurable gambling house defined on the Borel set $F$ and that for each $f$ in $F$

$$
\Gamma(f)=\left\{\gamma_{0}(f), \gamma_{1}(f), \ldots\right\}
$$

where $\gamma_{0}(f)=\delta(f)$ and each $\gamma_{n}$ is a measurable map from $F$ to $\mathscr{P}(F)$. $\Gamma$ is called a countable house for the sake of brevity. The utility function $u$ is assumed to be a bounded Borel function from $F$ to the reals in this section.

THEOREM 1. If $\Gamma$ is a countable house, then the functions $U_{n}$ for $n=0,1,2, \ldots$ and $U$ are Borel measurable. Also, $U_{\omega}=U$.

Proof. $U_{0}=u$ is measurable by assumption. Suppose $U_{n}$ is measurable. The maps $f \rightarrow \gamma_{k}(f) U_{n}$ are measurable since the $\gamma_{k}$ are measurable functions. Hence,

$$
U_{n+1}(f)=\sup _{k} \gamma_{k}(f) U_{n}
$$


is measurable. By induction, $U_{n}$ is measurable for $n=0,1,2, \ldots U_{\omega}=\lim U_{n}$ is measurable and, by Theorem $2.15 .5(\mathrm{~g})$ of [6], $U_{\omega}=U$.

The next result is analogous to Theorem 7(a) of [2].

THEOREM 2. Let $\Gamma$ be a countable house, $n$ be a positive integer, and $\varepsilon>0$. Then there is a measurable stationary family $\gamma^{\infty}$ available in $\Gamma$ such that

$$
u\left(\gamma^{\infty}(f)\right) \geqq U_{n}(f)-\varepsilon \text { for all } f \text { in } F .
$$

$\gamma$ may be chosen so that the map $f \rightarrow u\left(\gamma^{\infty}(f)\right)$ is Borel.

Proof. Construct $\gamma$ as in the proof of Theorem 1.1, using Theorem 1 of this section to assure that $\gamma$ is measurable.

The functions $u\left(\gamma^{\infty}(f), n\right)$ are measurable in $f$ since

$$
u\left(\gamma^{\infty}(f), 1\right)=\int u\left(f_{1}\right) d \gamma\left(f_{1} \mid f\right)
$$

and

$$
u\left(\gamma^{\infty}(f), n\right)=\int u\left(\gamma^{\infty}\left(f_{1}\right), n-1\right) d \gamma\left(f_{1} \mid f\right)
$$

for $n>1$. But $\gamma$ was constructed so that $u\left(\gamma^{\infty}(f)\right)=\lim u\left(\gamma^{\infty}(f), n\right)$.

LEMMA 1. Let $\varepsilon>0$ and $\varepsilon^{\prime}>0$. If $\Gamma$ is a countable house and $p$ is any probability measure on $\mathscr{B}(F)$, then there is available a measurable stationary family $\gamma^{\infty}$ such that $f \rightarrow u\left(\gamma^{\infty}(f)\right)$ is measurable and

$$
p\left\{f: u\left(\gamma^{\infty}(f)\right)>U(f)-\varepsilon\right\}>1-\varepsilon^{\prime} .
$$

Proof. By Theorem $1, U_{n} \rightarrow U$ pointwise as $n \rightarrow \infty$. Since the $U_{n}$ and $U$ are measurable, $U_{n} \rightarrow U$ in $p$-probability. Choose $n$ so that

$$
p\left\{f: U_{n}(f)>U(f)-\varepsilon / 2\right\}>1-\varepsilon^{\prime} .
$$

Then choose $\gamma^{\infty}$ by Theorem 2 so that $u\left(\gamma^{\infty}(f)\right)>U_{n}(f)-\varepsilon / 2$ for all $f$.

To complete the proof of Theorem 2.3 for the case of $\Gamma$ countable, we adapt some ingenious techniques due to Donald Ornstein. A similar adaptation was used by Barbosa-Dantas [1] for the case of positive dynamic programming.

Let $\varepsilon>0$ and assume, as we may, that $0 \leqq u \leqq 1$. Let $\varepsilon_{1}, \varepsilon_{2}, \ldots$ be small positive numbers whose size will be specified later.

Choose $\gamma_{1}^{\infty}$ by Lemma 1 such that

$$
p\left\{f: u\left(\gamma_{1}^{\infty}(f)\right)>U(f)-\varepsilon_{1}^{2}\right\}>\frac{1}{2}
$$

and define

$$
\begin{aligned}
& S_{1}=\left\{f: u\left(\gamma_{1}^{\infty}(f)\right)>U(f)-\varepsilon_{1}^{2}\right\}, \\
& T_{1}=\left\{f: u\left(\gamma_{1}^{\infty}(f)\right)>U(f)-\varepsilon_{1}\right\} .
\end{aligned}
$$


If $f \in S_{1}, \gamma_{1}^{\infty}(f)$ is a very good strategy even when the gambler is only allowed to use it on the set $T_{1}$. For example, if we set

$$
\begin{aligned}
\bar{\gamma}_{1}(f) & =\gamma_{1}(f), & & f \in T_{1}, \\
& =\delta(f), & & f \notin T_{1},
\end{aligned}
$$

then

$$
u\left(\bar{\gamma}_{1}^{\infty}(f)\right) \geqq u\left(\gamma_{1}^{\infty}(f)\right)-\varepsilon_{1} \quad \text { whenever } f \in S_{1} .
$$

To see this, apply Lemma 3.1 and Corollary 3.2.

Now consider a new gambling house $\Gamma_{1}$ given by

$$
\begin{aligned}
\Gamma_{1}(f) & =\Gamma(f) & & \text { if } f \notin T_{1}, \\
& =\left\{\gamma_{1}(f), \delta(f)\right\} & & \text { if } f \notin T_{1} .
\end{aligned}
$$

Notice $\Gamma_{1}$ is a countable gambling house and $\bar{\gamma}^{\infty}(f)$ is available in $\Gamma_{1}$ at any $f \in S_{1}$. Also, we have

LEMMA 2. $U_{1}(f) \geqq U(f)-\varepsilon_{1}$ for all $f \in F$.

Proof. This is clear if $f \in T_{1}$. Suppose $f \notin T_{1}$. Let $\varepsilon^{\prime}>0$ and choose a strategy $\sigma$ available at $f$ in $\Gamma$ such that $u(\sigma)>U(f)-\left(\varepsilon^{\prime}\right)^{2}$. Let $\bar{\sigma}$ be the composition of $\sigma$ with $\gamma_{1}^{\infty}$ at the time the gambler enters $T_{1}$. Then $\bar{\sigma}$ is available at $f$ in $\Gamma_{1}$. So by Lemma 3.2 ,

$$
U_{1}(f) \geqq u(\bar{\sigma})>U(f)-\left(\varepsilon^{\prime}\right)^{2}-\varepsilon^{\prime}-\varepsilon_{1} .
$$

Now we proceed to define a sequence of countable gambling houses $\Gamma_{1}, \Gamma_{2}, \ldots$ and measurable stationary families $\gamma_{1}^{\infty}, \gamma_{2}^{\infty}, \ldots$ inductively.

Suppose

$$
\begin{aligned}
S_{k} & =\left\{f: u\left(\gamma_{k}^{\infty}(f)\right)>U_{k-1}(f)-\varepsilon_{k}^{2}\right\}, \\
T_{k} & =\left\{f: u\left(\gamma_{k}^{\infty}(f)\right)>U_{k-1}(f)-\varepsilon_{k}\right\}, \\
\bar{\gamma}_{k}(f) & =\gamma_{k}(f), \quad f \in T_{k}, \\
& =\delta(f), \quad f \notin T_{k} .
\end{aligned}
$$

And suppose we have defined $\Gamma_{1}, \ldots, \Gamma_{k}$ and $\gamma_{1}^{\infty}, \ldots, \gamma_{k}^{\infty}$ so that for all $j=2, \ldots, k$

$$
U_{k} \geqq U_{k-1}-\varepsilon_{k},
$$

$$
\bar{\gamma}_{j}^{\infty}(f) \text { is available in } \Gamma_{k} \text { if } f \in S_{j} \text {, }
$$

and

$$
p\left(S_{j}\right) \geqq 1-\left(\frac{1}{2}\right)^{j} .
$$

Then choose $\gamma_{k+1}^{\infty}$ available in $\Gamma_{k}$ so that $p\left(S_{k+1}\right) \geqq 1-\left(\frac{1}{2}\right)^{k+1}$ and define

$$
\begin{aligned}
\Gamma_{k+1}(f) & =\Gamma_{k}(f) & & \text { if } f \notin T_{k+1} \text { or if } \gamma_{k+1}(f)=\delta(f), \\
& =\left\{\gamma_{k+1}(f), \delta(f)\right\} & & \text { if } f \in T_{k+1} \text { and } \gamma_{k+1}(f) \neq \delta(f) .
\end{aligned}
$$


The arguments used in the case of $\Gamma_{1}$ apply to show that properties (1)-(3) are preserved.

Now we define a house $\Gamma^{\prime}$ by

$$
\begin{aligned}
\Gamma^{\prime}(f) & =\bigcap_{k=1}^{\infty} \Gamma_{k}(f), \quad f \in \bigcup_{k=1}^{\infty} T_{k}, \\
& =\{\delta(f)\}, \quad f \notin \bigcup_{k=1}^{\infty} T_{k} .
\end{aligned}
$$

If $f \in S_{k}$ for some $k$, then $\bar{\gamma}_{k}^{\infty}(f)$ is available in $\Gamma^{\prime}$ and

$$
\begin{aligned}
U^{\prime}(f) & \geqq u\left(\bar{\gamma}_{k}^{\infty}(f)\right) \\
& \geqq u\left(\gamma_{k}^{\infty}(f)\right)-\varepsilon_{k} \\
& >U_{k-1}(f)-\varepsilon_{k}^{2}-\varepsilon_{k} \\
& \geqq U(f)-\varepsilon_{k}^{2}-\left(\varepsilon_{1}+\cdots+\varepsilon_{k}\right) .
\end{aligned}
$$

Choose the $\varepsilon_{k}$ so that $\sum_{1}^{\infty} \varepsilon_{k}+\sum_{1}^{\infty} \varepsilon_{k}^{2}<\varepsilon$. Then since $p\left(\bigcup S_{k}\right)=1$,

$$
p\left\{f: U^{\prime}(f)>U(f)-\varepsilon\right\}=1 .
$$

Notice also that $\Gamma^{\prime}$ is a measurable house having at most 2 gambles available at any fortune. That is,

$$
\Gamma^{\prime}(f)=\{\alpha(f), \delta(f)\}
$$

where $\alpha: F \rightarrow \mathscr{P}(F)$ is measurable. By Dubins and Savage [6, Theorem 3.9.5, p. 61] the measurable family $\gamma^{\infty}$ given by

$$
\begin{aligned}
\gamma(f) & =\alpha(f) & \text { if } u(f)<U^{\prime}(f), \\
& =\delta(f) & \text { if } u(f)=U^{\prime}(f),
\end{aligned}
$$

is a family of optimal strategies for $\Gamma^{\prime}$. Hence $u\left(\gamma^{\infty}(f)\right)=U^{\prime}(f)$ for all $f$ in $F$ and, by (4), Theorem 2,3 is now proved for $\Gamma$ a countable house.

5. Every measurable house is almost dominated by a countable house. If $\Gamma$ and $\Gamma^{\prime}$ are gambling houses on the same space of fortunes $F$ and $\Gamma(f) \supseteq \Gamma^{\prime}(f)$ for all $f$ in $F$, then $\Gamma^{\prime}$ is a subhouse of $\Gamma[6$, p. 123].

The main result of this section is

THEOREM 1. Let $\Gamma$ be a leavable, measurable house defined on $F$ and let $p$ be a probability measure on $\mathscr{B}(F)$. Then there is a countable subhouse $\Gamma^{\prime}$ of $\Gamma$ such that $U^{\prime}=U$ p-almost certainly, where $U^{\prime}$ and $U$ are the optimal return functions of $\Gamma^{\prime}$ and $\Gamma$ respectively.

The proof of Theorem 1 will be given in four lemmas. Theorem 1 and my proof of it are analogous to Theorem 2 of [2].

Throughout this section $\Gamma$ is assumed to be leavable and measurable.

A measurable family of strategies $\bar{\sigma}$ is a map from $F$ to measurable strategies such that the composition $f \rightarrow p(\bar{\sigma}(f))$ is measurable from $F$ to $\mathscr{P}(H)$. 
LEMMA 1. Let $p \in \mathscr{P}(F), n$ be a positive integer, and $\varepsilon>0$. Then there is available in $\Gamma$ a measurable family of strategies $\bar{\sigma}$ such that

$$
p\left\{f: u(\bar{\sigma}(f), n)>U_{n}(f)-\varepsilon\right\}=1 .
$$

Proof. Same as that of the inductive step in Theorem 2.2.

The next lemma generalizes slightly the first half of the lemma in [2].

LEMMA 2. Let $X$ and $Y$ be Borel sets, $v$ a bounded Borel function from $X \times Y$ to the reals, and $q$ a regular conditional distribution on $Y$ given $X$. Then there is $a$ Borel function $g$ from $X$ to $Y$ such that

$$
v(x, g(x)) \geqq \int v(x, y) d q(y \mid x)
$$

for all $x$ in $X$.

If $\Gamma$ is a Borel subset of $X \times Y, p$ is a probability measure on the Borel subsets of $X$, and $p\left\{x: q\left(\Gamma_{x} \mid x\right)=1\right\}=1$, then $g$ can be chosen to have the additional property

$$
p\left\{x: g(x) \in \Gamma_{x}\right\}=1 .
$$

Proof. The first half of the lemma is the same as in [2]. So assume $g$ satisfies (1). Let

$$
S=\left\{(x, y): v(x, y) \geqq \int v(x, y) d q(y \mid x)\right\} .
$$

Then $q\left(S_{x} \cap \Gamma_{x} \mid x\right)>0$ if $x \notin B$ where $B \in \mathscr{B}(X)$ and $p(B)=0$. By Theorem 2 of [4], there is a measurable map $\bar{g}: X \sim B \rightarrow Y$ such that $\bar{g}(x) \in S_{x} \cap \Gamma_{x}$ for all $x \in X \sim B$. Define $\bar{g}(x)=g(x)$ if $x \in B$. Then $\bar{g}$ satisfies (1) and (2).

LEMMA 3. Let $\bar{\sigma}$ be a measurable family of strategies available in $\Gamma$. If $u$ is any bounded, Borel map from $F$ to the reals, and $p \in \mathscr{P}(F)$, then there is a measurable function $\gamma: F \rightarrow \mathscr{P}(F)$ such that $\gamma(f) \in \Gamma(f)$ for all $f$ and

$$
\int u\left(f_{n}\right) d \bar{\sigma}\left(f_{n} \mid f\right) d p(f) \leqq \int u\left(f_{n}\right) d \gamma\left(f_{n} \mid f_{n-1}\right) d \bar{\sigma}\left(f_{n-1} \mid f\right) d p(f)
$$

where $\bar{\sigma}\left(f_{k} \mid f\right)$ is the marginal distribution of $f_{k}$ under $\bar{\sigma}(f)$.

The intuitive meaning of the lemma is that a gambler with fortune $f$, who gambles for $n$-days using the strategy $\bar{\sigma}(f)$ and is paid $u\left(f_{n}\right)$, can expect to do as well by using $\bar{\sigma}(f)$ for the first $n-1$ days and then using a gamble $\gamma\left(f_{n-1}\right)$ which depends only on his fortune at the $(n-1)$ th day.

Proof. Let $Q$ be that probability on $F \times H$ whose marginal distribution on $F$ is $p$ and whose conditional distribution on $H$ given $f$ is $p(\bar{\sigma}(f))$. Consider

$$
\bar{\sigma}(f)_{n-1}\left(f_{1}, \ldots, f_{n-1}\right)
$$


as a random variable mapping $F^{n}$ to $\mathscr{P}(F)$. Let $q$ be a version of its conditional $Q$-distribution given $f_{n-1}$, and let $\mu$ denote the $Q$-marginal distribution of $f_{n-1}$. Then

$$
\int u\left(f_{n}\right) d \bar{\sigma}\left(f_{n} \mid f\right) d p(f)=\int u\left(f_{n}\right) d \gamma\left(f_{n}\right) d q\left(\gamma \mid f_{n-1}\right) d \mu\left(f_{n-1}\right) .
$$

Define $v(f, \gamma)=v(\gamma)=\int u\left(f_{n}\right) d \gamma\left(f_{n}\right)$. By Lemma 2 there is a measurable map $\bar{\gamma}$ from $F$ to $\mathscr{P}(F)$ such that

and

$$
v(\bar{\gamma}(f)) \geqq \int v(\gamma) d q(\gamma \mid f) \text { for all } f
$$

$$
\mu\{f: \bar{\gamma}(f) \in \Gamma(f)\}=1 .
$$

The desired function $\gamma$ is then given by

$$
\begin{array}{rlrl}
\gamma(f) & =\bar{\gamma}(f) & & \text { if } \bar{\gamma}(f) \in \Gamma(f), \\
& =\delta(f) & \text { if } \bar{\gamma}(f) \notin \Gamma(f) .
\end{array}
$$

A family of strategies $\bar{\gamma}$ is said to be Markov if there is a sequence $\gamma_{0}, \gamma_{1}, \ldots$ of gamble-valued functions on $F$ such that, for each $f$ in $F$ and every partial history $\left(f_{1}, f_{2}, \ldots, f_{n}\right)$,

$$
\bar{\gamma}(f)_{0}=\gamma_{0}(f), \quad \bar{\gamma}(f)_{n}\left(f_{1}, \ldots, f_{n}\right)=\gamma_{n}\left(f_{n}\right) .
$$

$\bar{\gamma}$ is measurable if the $\gamma_{n}$ can be taken to be measurable functions from $F$ to $\mathscr{P}(F)$.

Lemma 4. Let $p \in \mathscr{P}(F), n$ be a positive integer, and $\varepsilon>0$. There is available in $\Gamma$ a measurable Markov family of strategies $\bar{\gamma}$ such that

$$
p\left\{f: u(\bar{\gamma}(f), n) \geqq U_{n}(f)-\varepsilon\right\} \geqq 1-\varepsilon .
$$

Proof. Choose a measurable family of strategies $\bar{\sigma}$ by Lemma 1 so that

$$
p\left\{f: u(\bar{\sigma}(f), n) \geqq U_{n}(f)-\varepsilon^{2}\right\}=1 .
$$

Apply Lemma $3 n$-times to construct measurable maps $\gamma_{0}, \ldots, \gamma_{n-1}$ of $F$ to $\mathscr{P}(F)$ so that

$$
\int u(\bar{\gamma}(f), n) d p(f) \geqq \int u(\bar{\sigma}(f), n) d p(f)
$$

if $\bar{\gamma}$ is any Markov family whose first $n$ functions are the $\gamma_{k}$. Then

$$
\int u(\bar{\gamma}(f), n) d p(f) \geqq \int U_{n}(f) d p(f)-\varepsilon^{2},
$$

which implies (3).

To complete the proof of Theorem 1, choose measurable Markov families $\bar{\gamma}_{1}, \bar{\gamma}_{2}, \ldots$ such that

$$
p\left\{f: u\left(\bar{\gamma}_{n}(f), n\right) \geqq U_{n}(f)-1 / n\right\} \geqq 1-1 / n
$$


for $n=1,2, \ldots$ Then let $\Gamma^{\prime}$ be any countable subhouse of $\Gamma$ in which the $\bar{\gamma}_{n}$ are available.

Theorem 2.3 now follows from Theorem 1 and the result of $\S 4$.

Acknowledgement. I wish to express my deep appreciation to my advisor Lester Dubins for his guidance and sympathetic advice during the writing of this thesis. Also, I want to thank David Blackwell and David Freedman for several helpful conversations about dynamic programming and measure theory.

\section{REFERENCES}

1. C. A. Barbosa-Dantas, The existence of stationary optimal plans, Dissertation, Univ. of California, Berkeley, 1966.

2. David Blackwell, Discounted dynamic programming, Ann. Math. Statist. 36 (1965), 226-235.

3. — Positive dynamic programming, mimeographed, Univ. of California, Berkeley, 1965.

4. David Blackwell and C. Ryll-Nardzewski, Non-existence of everywhere proper conditional distributions, Ann. Math. Statist. 34 (1963), 223-225.

5. Lester Dubins and David Freedman, Measurable sets of measures, Pacific J. Math. 14 (1965), 1121-1222.

6. L. E. Dubins and L. J. Savage, How to gamble if you must, McGraw-Hill, New York, 1965.

7. Casimir Kuratowski, Topologie, Vol. 1, 4th ed., Monogr. Mat., PWN, Warsaw, 1958.

8. G. W. Mackey, Borel structures in groups and their duals, Trans. Amer. Math. Soc. 85 (1957), 134-165.

9. Pierre Novikoff, Sur les fonctions implicites mesurables B, Fund. Math. 17 (1931), 8-25.

10. Donald Ornstein, On the existence of stationary optimal strategies, mimeographed, Stanford Univ., Stanford, Calif., 1967.

11. R. E. Strauch, Measurable gambling houses, Trans. Amer. Math. Soc. 126 (1967), 64-72.

12. - Negative dynamic programming, Ann. Math. Statist. 37 (1966), 871-890.

\section{UNIVERSITY OF CALIFORNIA, Berkeley, California}

